\title{
Estimación del riesgo cardiovascular por composición corporal total
}

\author{
Óscar Medina ${ }^{1}$, Juan Manuel Sarmiento², Larry Quinn ${ }^{3}$, Sonia Merlano ${ }^{4}$, Fabián Antonio \\ Dávila ${ }^{5}$, Andrés Felipe Barragán ${ }^{6}$, Antonio José Lewis ${ }^{7}$, Iván René Mogollón, María José Pareja.
}

${ }^{1}$ Médico Internista, Endocrinólogo, Fundación Clínica Shaio,

Bogotá, Colombia.

${ }^{2}$ Médico del deporte, Fundación Clínica Shaio, Bogotá, Colombia.

${ }^{3}$ Residente de medicina del deporte, Universidad del Bosque,

Bogotá, Colombia.

${ }^{4}$ Médica Nuclear, Fundación Clínica Shaio, Bogotá, Colombia.

${ }^{5}$ Bioestadístico, Fundación Clínica Shaio, Bogotá, Colombia.

${ }^{6}$ Médico general, Universidad de la Sabana, Bogotá, Colombia.

${ }^{7}$ Médico general, Universidad de la Sabana, Bogotá, Colombia.

${ }^{8}$ Médico general, Universidad de la Sabana, Bogotá, Colombia.

Autor de correspondencia: Oscar Leonardo Medina Espitia

Correo: oscar.medina@shaio.org

Fecha de recepción: 31/10/2016

Fecha de aceptación: 19/12/2016

\section{Resumen}

$I$ ntroducción: La obesidad y la adiposidad están relacionadas con el aumento del riesgo cardiovascular. El índice de masa corporal (IMC) y el perímetro abdominal son las variables antropométricas más utilizadas para evaluar su magnitud. El presente estudio busca establecer la relación entre desenlaces cardiometabólicos y la adiposidad medida con Absorciometría Dual por rayos X (DXA), así como el rendimiento diagnóstico de la misma contra la medición de las variables antropométricas convencionales.

Materiales y métodos: Se realizó un estudio observacional de corte transversal; se calcularon las variables antropométricas y de composición corporal para 60 pacientes en programa de rehabilitación cardiaca fase II.

Resultados: Existió mayor prevalencia de obesidad por IMC $y$ adiposidad en mujeres que en hombres ( $p=0,01$ y 0,048$)$. La curva ROC encontró que el rendimiento del perímetro abdominal es solo 65\% y el del IMC del 65,6\% para el diagnóstico de adiposidad. Se encontraron relaciones significativas entre porcentaje de masa grasa elevado y la enfermedad coronaria (OR: 1,9 p= 0,042); el IMC aumentado con la hipertensión arterial (OR: 3,0 p= 0,0334) y el LDL > $70 \mathrm{mg} / \mathrm{dl}$ (OR: 0,4 p= 0,0178); el perímetro abdominal aumentado con la falla cardiaca (OR: 0,58 p=0,0382); la TMB baja con la hipertensión arterial (OR: $1,70 \mathrm{p}=0,046)$ y finalmente el IIRME disminuido con el LDL $>70 \mathrm{mg} / \mathrm{dl}$ y la falla cardiaca (OR: 0,4 p=0,0178y OR 1,96 p=0,078, respectivamente).
Conclusiones: La suma de la medición de las variables antropométricas y de composición corporal por DXA ofrece información valiosa para el estudio y estimación del riesgo cardiovascular y metabólico de los pacientes.

Palabras clave: Obesidad; DEXA Scans; Enfermedad Coronaria; Composición Corporal; Índice de Masa Corporal; Adiposidad.

\section{Abstract}

Introduction: Obesity and adiposity are associated with increased cardiovascular risk. The body mass index (BMI) and waist circumference are the most anthropometric variables used to assess their magnitude. This study aims to establish the relationship between adiposity and cardiometabolic outcomes measured by Dual X-ray Absorptiometry (DXA) as well as the diagnostic performance of the latter against the measurement of the conventional anthropometric variables.

Materials and methods: An observational cross-sectional study was conducted; anthropometric and body composition variables for 60 patients in cardiac rehabilitation program phase II were calculated.

Results: There was a higher prevalence of obesity by BMI and adiposity in women than in men ( $p=0.01$ and 0.048). The ROC curve found that the performance is only $65 \%$ for waist circumference and $65.6 \%$ for BMI for the diagnosis of adiposity. Significant correlations between high percentage of fat mass and coronary heart disease (OR: $1.9 p=0.042$ ) were found; as well as for increased BMI with hypertension (OR: $3.0 p=0.0334$ ) and $L D L>70 \mathrm{mg} / \mathrm{dl}$ (OR: $0.4 p=0.0178$ ); increased waist circumference with heart failure (OR: $0.58 p=0.0382$ ); low basal metabolic rate (BMR) with hypertension (OR: $1.70 p=0.046$ ) and finally the decreased fat free mass index (FFMI) with $L D L>$ $70 \mathrm{mg} / \mathrm{dl}$ and heart failure (OR: $0.4 p=0.0178$ and $\mathrm{OR}: 1.96 p=$ 0.078 respectively).

Conclusions: The addition of body composition variables by DXA and anthropometric variables, provides valuable information for the study and estimation of cardiovascular and metabolic risk.

Key Words: Obesity; DEXA Scans; Coronary Disease; Body Composition; Body Mass Index; Adiposity. 


\section{Introducción}

La incidencia de obesidad se ha duplicado en las últimas tres décadas ${ }^{(1)}$. El índice de masa corporal (IMC) ha sido la medición más utilizada tradicionalmente, definiendo como obesos a los individuos con valores $>30 \mathrm{~kg} / \mathrm{m}^{2}$. Esta misma es la que se ha utilizado para la estimación de la prevalencia global y para valorar el impacto de esta enfermedad en los desenlaces metabólicos y cardiovasculares. Sin embargo, se trata de una herramienta que utiliza la relación de peso/talla, sin tener en cuenta los componentes corporales, limitando su utilidad como indicador de adiposidad ${ }^{(2-4)}$; por lo cual, en la última década se ha venido utilizando la medición de variables como la masa magra como marcador de sarcopenia ${ }^{(5)}$ y la masa grasa, mediante técnicas como Absorciometría Dual por rayos $\mathrm{X}$ $(\mathrm{DXA})^{(6-8)}$, la cual realiza una cuantificación precisa, siendo una alternativa atractiva al cálculo del IMC.

El objetivo del presente estudio fue identificar la relación entre las variables antropométricas (IMC y perímetro abdominal) y de composición corporal (porcentaje de masa grasa y magra), con los desenlaces cardiovasculares en pacientes de rehabilitación cardiaca fase II.

\section{Materiales y métodos}

Se realizó un estudio observacional de corte transversal; se extrajeron los registros del programa de seguimiento y control de pacientes en rehabilitación cardiaca fase II. Se describieron las características generales y se exploraron diferencias entre las variables determinantes de la composición corporal, de riesgo y desenlaces cardiovasculares. Las variables cuantitativas se dicotomizaron según los puntos de corte de riesgo, definidos en la literatura como normales y anormales, así, los riesgos se definieron en IMC $>30 \mathrm{~kg} /$ $\mathrm{m}^{2}$, perímetro abdominal $>90 \mathrm{~cm}, \mathrm{LDL}>70 \mathrm{mg} / \mathrm{dl}$, colesterol total $>200 \mathrm{mg} / \mathrm{dl}$, triglicéridos $>150 \mathrm{mg} / \mathrm{dl}$, glucemia $>125$ $\mathrm{mg} / \mathrm{dl}, \mathrm{HDL}<40 \mathrm{mg} / \mathrm{dl}$ en mujeres y $<50 \mathrm{mg} / \mathrm{dl}$ en hombres. Porcentaje de masa grasa $>30 \%$ para hombres y $>35 \%$ para mujeres, porcentaje de masa magra $<60 \%$ en hombres y $<40 \%$ en mujeres, relación androide/ginecoide $>1$ en hombres y $>0,8$ en mujeres; tasa metabólica basal (TMB) $<1,200$ $\mathrm{kcal} /$ día (sedentario), índice relativo de músculo esquelético (IRME) $<7,26$ en hombres y $<5,46$ en mujeres, mediante la utilización de radiodensitómetro óseo lunar iDXA con software Encore $\mathrm{e}^{(4,5,9-11)}$.

Las variables cualitativas fueron descritas con frecuencias absolutas y relativas, las cuantitativas con medias e intervalos de confianza del 95\% o medianas y rangos intercuartílicos (dependiendo de la distribución); las diferencias entre variables categóricas se exploraron por tablas de contingencia y Chi cuadrado; entre variables categóricas y numéricas se exploraron por U Mann Whitney. Todos los contrastes asumieron un $\mu$ (nivel de significancia) inferior al 0,05.

\section{Resultados}

Se revisaron 60 registros de pacientes del programa de rehabilitación cardiaca, el 41,1\% de los casos fueron mujeres, la mediana de edad fue de 66 años, sin diferencias significativas por sexo ( $p=0,583)$ (figura 1 ).

La mayoría de los pacientes fueron hipertensos $(69,5 \%)$, tenían antecedente de enfermedad coronaria (90\%) y de angioplastia $(58,3 \%)$, perímetro abdominal $>90 \mathrm{~cm}(59,6 \%)$, porcentaje de masa grasa elevado $(72,9 \%)$ y porcentaje de masa magra normal $(96,6 \%)$; todos tenían una relación androide/ginecoide elevada. Se apreciaron diferencias significativas por sexo para las variables: IMC, colesterol total, HDL, LDL, triglicéridos, TMB y porcentaje de masa grasa (tabla 1). Adicionalmente, existió mayor prevalencia de obesidad por IMC y porcentaje de masa grasa en mujeres que en hombres, con significancia estadística en el último parámetro.

Figura 1. Pirámide poblacional (edad y género)

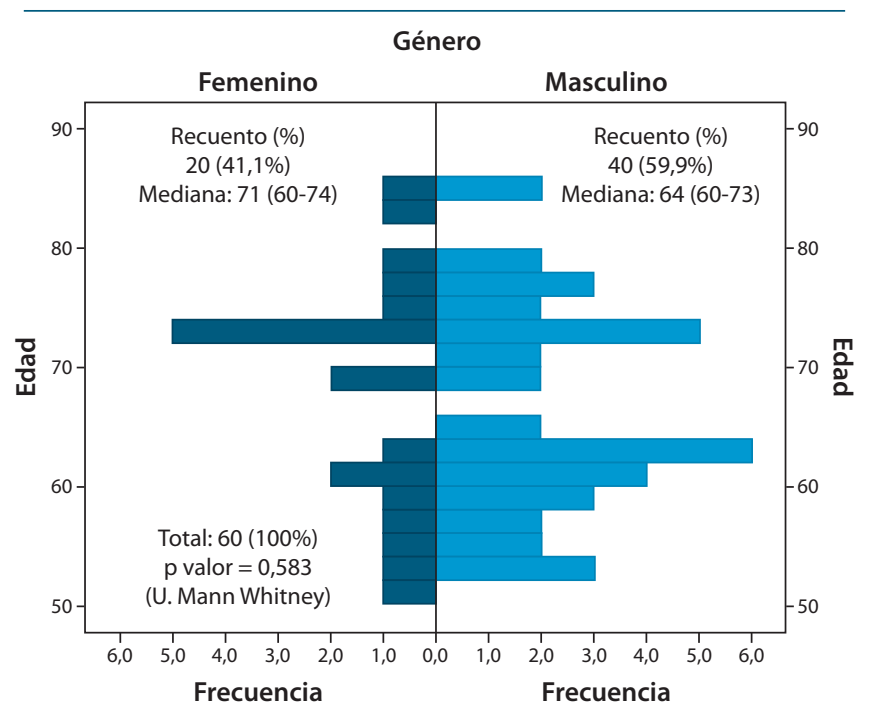

Figura 2. Relación entre enfermedad coronaria y porcentaje de masa grasa elevado

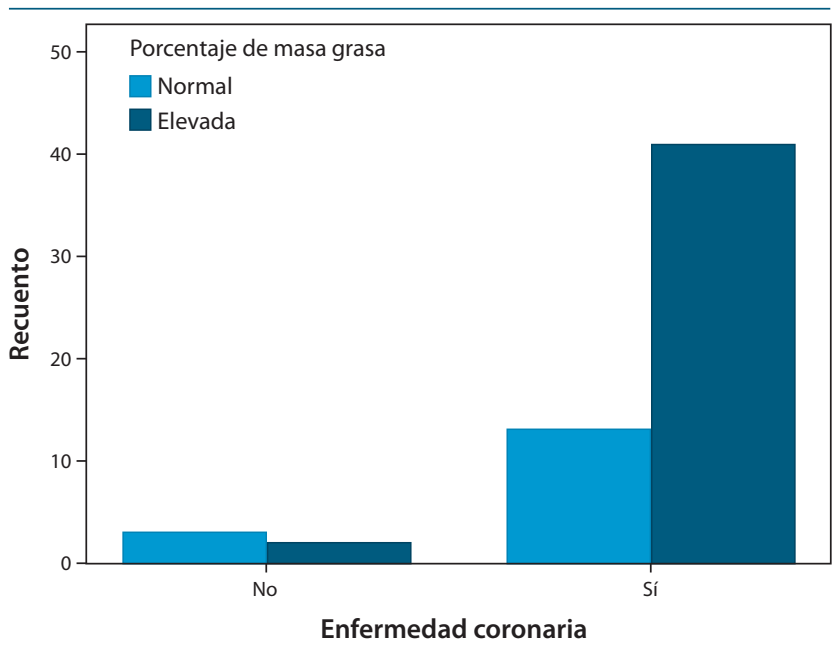


La mayoría de los pacientes que presentaron enfermedad coronaria tuvieron porcentaje de masa grasa elevado $(75,9 \%)$ $(\mathrm{p}=0,84)$ (tabla 2 y figura 2 ).

Se encontró relación entre el porcentaje de masa grasa aumentado con perímetro abdominal e IMC aumentados ( $\mathrm{p}=$ 0,039 y 0,028 respectivamente), el resto de asociaciones no fueron estadísticamente significativas (tabla 3).

Se exploró en curva ROC la relación entre las variables de la composición corporal, encontrando un área bajo la curva (rendimiento diagnóstico) moderada para el perímetro abdo- minal (AUC: 65\%) y el IMC (AUC: 65,6\%) (p valor de 0,942; 0,082 y 0,070 respectivamente) (figura 3).

En la exploración de Odds Ratios, se encontraron relaciones significativas entre porcentaje de masa grasa elevado y la enfermedad coronaria (OR: 1,9 p = 0,042); el IMC aumentado con la hipertensión arterial (OR: 3,0 p = 0,0334) y el LDL > 70 mg/dl (OR: 0,4 p = 0,0178); el perímetro abdominal aumentado con la falla cardiaca (OR: 0,58 $p=0,0382$ ); la TMB baja con la hipertensión arterial (OR: 1,70 p=0,046) y, finalmente, el IIRME disminuido con el LDL $>70 \mathrm{mg} / \mathrm{dl}$ y la falla cardiaca (OR: 0,4 p=0,0178 y OR: $1,96 \mathrm{p}=0,078$ respectivamente).

Tabla 1. Características generales

\begin{tabular}{|c|c|c|c|c|}
\hline \multirow[b]{2}{*}{ Variable } & \multicolumn{2}{|c|}{ Género } & \multirow{2}{*}{$\begin{array}{c}\text { Total } \\
\text { N 60: } \\
(100 \%)\end{array}$} & \multirow{2}{*}{$\begin{array}{c}\text { p. valor } \\
\text { (chi cuadrado) }\end{array}$} \\
\hline & $\begin{array}{l}\text { Femenino: } \\
20(41,1 \%)\end{array}$ & $\begin{array}{l}\text { Masculino: } \\
40(59,9 \%)\end{array}$ & & \\
\hline Hipertensión Arterial Sistémica & $16(84,2)$ & $25(62,5)$ & $41(69,5)$ & 0,091 \\
\hline Tabaquismo & $2(10)$ & $4(10)$ & $6(10)$ & 1 \\
\hline Infarto agudo de miocardio & $10(50)$ & $20(50)$ & $30(50)$ & 1 \\
\hline Falla cardiaca & $4(20)$ & $10(25)$ & $14(23,3)$ & 0,666 \\
\hline Enfermedad arterial periférica & $1(5)$ & $0(0)$ & $1(1,7)$ & 0,154 \\
\hline Accidente cerebrovascular & $2(10)$ & $1(2,5)$ & $3(5)$ & 0,209 \\
\hline Diabetes mellitus & $7(35)$ & $8(20)$ & $15(25)$ & 0,206 \\
\hline Valvulopatía & $2(10)$ & $3(7,5)$ & $5(8,3)$ & 0,741 \\
\hline Enfermedad coronaria & $18(90)$ & $36(90)$ & $54(90)$ & 1 \\
\hline Angioplastia & $10(50)$ & $25(62,5)$ & $35(58,3)$ & 0,355 \\
\hline Revascularización miocárdica & $5(25)$ & $13(32,5)$ & $18(30)$ & 0,55 \\
\hline Hipertensión pulmonar & $0(0)$ & $4(10)$ & $4(6,7)$ & 0,143 \\
\hline Valvuloplastia & $2(10)$ & $3(7,5)$ & $5(8,3)$ & 0,741 \\
\hline Revascularización periférica & $0(0)$ & $1(2,5)$ & $1(1,7)$ & 0,476 \\
\hline Marcapasos & $2(10)$ & $1(2,5)$ & $3(5)$ & 0,209 \\
\hline Cardiodesfibrilador & $0(0)$ & $4(10)$ & $4(6,7)$ & 0,143 \\
\hline $\mathrm{IMC}>30 \mathrm{~kg} / \mathrm{m}^{2}$ & $11(55)$ & $6(15)$ & $17(25,4)$ & 0,01 \\
\hline Nivel de actividad física: activo/a & $4(20)$ & $21(52,5)$ & $25(41,7)$ & 0,016 \\
\hline Colesterol $>200 \mathrm{mg} / \mathrm{dl}$ & $7(36,8)$ & $5(13,5)$ & $12(21,4)$ & 0,044 \\
\hline HDL normal & $8(40)$ & $4(10)$ & $12(20)$ & 0,006 \\
\hline $\mathrm{LDL}>70 \mathrm{mg} / \mathrm{dl}$ & $14(70)$ & $20(50)$ & $34(56,7)$ & 0,003 \\
\hline Triglicéridos $>150 \mathrm{mg} / \mathrm{dl}$ & $9(52,9)$ & $14(37,8)$ & $23(42,6)$ & 0,297 \\
\hline Glucemia >125 gr/dl & $6(33,3)$ & $7(21,9)$ & $13(26)$ & 0,375 \\
\hline $\mathrm{HbA} 1 \mathrm{C}>6,5 \%$ & $2(20)$ & $6(25)$ & $8(23,5)$ & 0,754 \\
\hline Perímetro abdominal $>90 \mathrm{~cm}$ & $11(57,9)$ & $23(60,5)$ & $34(59,6)$ & 0,849 \\
\hline Porcentaje de masa grasa elevada & $17(89,5)$ & $26(65)$ & $43(72,9)$ & 0,048 \\
\hline Porcentaje de masa magra disminuido & $0(100)$ & $2(5)$ & $2(3,4)$ & 0,983 \\
\hline Tasa metabólica en reposo disminuida (<1,200kcal/día) & $15(75)$ & $2(5)$ & $17(28,3)$ & 0,000 \\
\hline Relación androide/ginecoide elevada & $19(100)$ & $39(100)$ & $58(100)$ & --- \\
\hline
\end{tabular}


Tabla 2. Relación entre enfermedad coronaria y porcentaje de masa grasa elevado

\begin{tabular}{|c|c|c|c|c|}
\hline \multirow{2}{*}{\multicolumn{2}{|c|}{ Variable }} & \multicolumn{3}{|c|}{ Porcentaje de masa grasa } \\
\hline & & Normal (\%) & Elevada (\%) & Total (\%) \\
\hline \multirow{2}{*}{$\begin{array}{l}\text { Enfermedad } \\
\text { coronaria }\end{array}$} & No & $3(60)$ & $2(40)$ & $5(100)$ \\
\hline & Sí & $13(24,1)$ & $41(75,9)$ & $54(100)$ \\
\hline & & $16(27)$ & $43(73)$ & $59(100)$ \\
\hline
\end{tabular}

Chi cuadrado de Person (valor de $\mathrm{p}$ ) $=0,84$

Tabla 3. Asociación porcentaje de masa grasa vs. perímetro abdominal, IMC, tasa metabólica, índice relativo de músculo esquelético

\begin{tabular}{l|c|c|c|c}
\hline \multicolumn{2}{|c|}{ Variable } & \multicolumn{2}{c|}{$\begin{array}{c}\text { Porcentaje de } \\
\text { masa grasa }\end{array}$} & $\begin{array}{c}\text { Chi } \\
\text { cuadrado } \\
\text { (valor de p) }\end{array}$ \\
\cline { 2 - 4 } & Normal & Elevada & Nán \\
\cline { 1 - 4 } $\begin{array}{l}\text { Perímetro } \\
\text { abdominal }\end{array}$ & $>90 \mathrm{~cm}$ & 6 & 27 & 0,039 \\
\hline IMC & $>30 \mathrm{~kg} / \mathrm{m}^{2}$ & 1 & 15 & $0,028^{*}$ \\
\hline $\begin{array}{l}\text { Tasa } \\
\text { metabólica } \\
\text { en reposo }\end{array}$ & $\begin{array}{c}\text { Disminuida } \\
(<1,200 \mathrm{kcal} / \\
\text { dia) }\end{array}$ & 3 & 13 & $0,778^{*}$ \\
\hline $\begin{array}{l}\text { Índice relativo } \\
\text { de músculo } \\
\text { esquelético } \\
\text { (kg/m }{ }^{2} \text { ) }\end{array}$ & $\begin{array}{r}\text { Disminuido } \\
\hline\end{array}$ & 5 & 9 & $0,407^{*}$ \\
\hline
\end{tabular}

*Recuentos inferiores a 5 individuos en una de las casillas, los resultados de Chicuadrado pueden no ser válidos.

Figura 3. Curva ROC: IMC vs. Perímetro abdominal para diagnóstico de adiposidad (porcentaje de masa grasa aumentado)

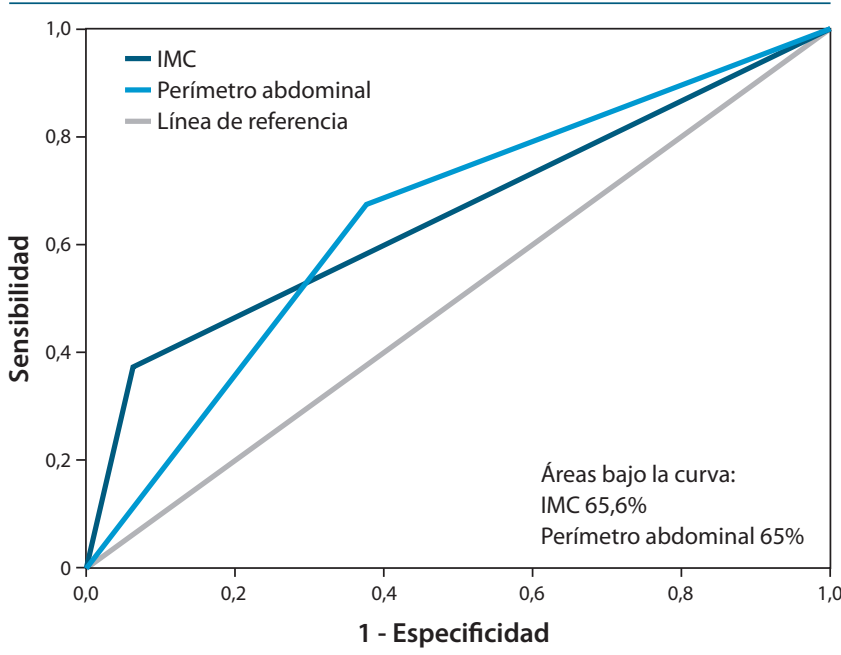

Tabla 4. Odds Ratio comparados de las variables de composición corporal

\begin{tabular}{|c|c|c|}
\hline Variable & OR (IC 95\%) & $\begin{array}{c}\text { Valor de } p \\
\text { (a } 1 \text { cola) }\end{array}$ \\
\hline \multicolumn{3}{|c|}{ Porcentaje grasa corporal aumentado } \\
\hline $\mathrm{LDL}>70 \mathrm{mg} / \mathrm{dl}$ & $0,75(1,02 ; 0,55)$ & 0,360 \\
\hline Colesterol total $>200 \mathrm{mg} / \mathrm{dl}$ & $0,73(1,28 ; 0,41)$ & 0,091 \\
\hline Hipertensión arterial & $1,44(2,24 ; 0,93)$ & 0,270 \\
\hline Diabetes mellitus & $1,27(1,69 ; 0,96)$ & 0,082 \\
\hline Triglicéridos $>150 \mathrm{mg} / \mathrm{dl}$ & $0,79(1,15 ; 0,54)$ & 0,094 \\
\hline Falla cardiaca & $1,07(1,52 ; 0,76)$ & 0,355 \\
\hline Accidente cerebrovascular & $0,91(2,06 ; 0,4)$ & 0,402 \\
\hline Enfermedad coronaria & $1,9(5,62 ; 0,64)$ & 0,042 \\
\hline \multicolumn{3}{|l|}{ IMC aumentado } \\
\hline $\mathrm{LDL}>70 \mathrm{mg} / \mathrm{dl}$ & $0,417(0,979 ; 0,178)$ & 0,017 \\
\hline Colesterol total $>200 \mathrm{mg} / \mathrm{dl}$ & $0,524(1,99 ; 0,13)$ & 0,151 \\
\hline Hipertensión arterial & $3,073(12,14 ; 0,77)$ & 0,033 \\
\hline Diabetes mellitus & $0,643(1,93 ; 0,21)$ & 0,204 \\
\hline Triglicéridos $>150 \mathrm{mg} / \mathrm{dl}$ & $0,539(1,50 ; 0,19)$ & 0,108 \\
\hline Falla cardiaca & $0,704(2,10 ; 0,23)$ & 0,256 \\
\hline Accidente cerebrovascular & $1,188(6,20 ; 0,22)$ & 0,421 \\
\hline Enfermedad coronaria & $0,519(1,29 ; 0,20)$ & 0,107 \\
\hline \multicolumn{3}{|c|}{ Perímetro abdominal aumentado } \\
\hline $\mathrm{LDL}>70 \mathrm{mg} / \mathrm{dl}$ & $0,78(1,19 ; 0,51)$ & 0,128 \\
\hline Colesterol total $>200 \mathrm{mg} / \mathrm{dl}$ & $0,72(1,44 ; 0,36)$ & 0,148 \\
\hline Hipertensión arterial & $1,25(2,16 ; 0,72)$ & 0,195 \\
\hline Diabetes mellitus & $1,11(1,76 ; 0,69)$ & 0,342 \\
\hline Triglicéridos $>150 \mathrm{mg} / \mathrm{dl}$ & $1,04(1,66 ; 0,65)$ & 0,430 \\
\hline Falla cardiaca & $0,58(1,2 ; 0,28)$ & 0,038 \\
\hline Enfermedad coronaria & $1,22(2,79 ; 0,53)$ & 0,305 \\
\hline \multicolumn{3}{|l|}{ TMB disminuida } \\
\hline $\mathrm{LDL}>70 \mathrm{mg} / \mathrm{dl}$ & $0,74(1,13 ; 0,48)$ & 0,200 \\
\hline Colesterol total $>200 \mathrm{mg} / \mathrm{dl}$ & $0,4(1,05 ; 0,15)$ & 0,064 \\
\hline Hipertensión arterial & $1,70(0,94 ; 0,52)$ & 0,046 \\
\hline Diabetes mellitus & $1,08(2,94 ; 0,40)$ & 0,869 \\
\hline Triglicéridos $>150 \mathrm{mg} / \mathrm{dl}$ & $0,87(1,69 ; 0,45)$ & 0,707 \\
\hline Falla cardiaca & $0,71(1,81 ; 0,27)$ & 0,483 \\
\hline Accidente cerebrovascular & $0,19(2,03 ; 0,19)$ & 0,131 \\
\hline Enfermedad coronaria & $1,02(1,25 ; 0,84)$ & 0,774 \\
\hline \multicolumn{3}{|l|}{ IRMM disminuido } \\
\hline $\mathrm{LDL}>70 \mathrm{mg} / \mathrm{dl}$ & $0,417(0,979 ; 0,178)$ & 0,017 \\
\hline Colesterol total $>200 \mathrm{mg} / \mathrm{dl}$ & $0,33(2,3 ; 0,05)$ & 0,102 \\
\hline Hipertensión arterial & $0,81(2,07 ; 0,32)$ & 0,332 \\
\hline Diabetes mellitus & $0,8(2,49 ; 0,26)$ & 0,347 \\
\hline Triglicéridos $>150 \mathrm{mg} / \mathrm{dl}$ & $0,84(2,24 ; 0,32)$ & 0,365 \\
\hline Falla cardiaca & $1,96(4,85 ; 0,8)$ & 0,078 \\
\hline Enfermedad coronaria & $0,56(1,81 ; 0,17)$ & 0,185 \\
\hline
\end{tabular}




\section{Discusión}

La adiposidad (porcentaje de masa grasa aumentado) se correlaciona con disregulación cardiometabólica y aumento del riesgo cardiovascular ${ }^{(12-14)}$.

En nuestro estudio, el IMC se encontró aumentado solo en el $25,4 \%$ de los pacientes, la adiposidad se presentó en una cantidad mayor (72,9\%), lo que concuerda con los hallazgos de otros autores en donde se evidencia que el IMC subestima el diagnóstico de obesidad, principalmente en hombres y en ancianos, lo que podría explicarse porque éstos fueron realizados en población anglosajona (blanca) ${ }^{(3)}$ Pasco et al. encontraron que el 29,5\% de los pacientes masculinos y el $24,7 \%$ de los femeninos eran obesos mediante porcentaje de masa grasa, mientras que solo el $19,7 \%$ y el $28,2 \%$, respectivamente, fueron obesos de acuerdo con el IMC. La inhabilidad de distinguir las contribuciones a la masa corporal de los distintos componentes de la composición corporal es probablemente la razón por la cual la medición de la adiposidad se puede ver falseada en el $\operatorname{IMC}^{(3,15)}$.

Adicionalmente, la obesidad fue más prevalente en mujeres que en hombres y se observaron hallazgos similares para los porcentajes de grasa corporal medidos por DXA, ambos con significancia estadística. Otros estudios ya habían demostrado resultados similares ${ }^{(16-18)}$ y la teoría más aceptada es el cambio hormonal que sucede en las mujeres posmenopáusicas, condicionando fenómenos que favorecen la pérdida de masa magra y la acumulación de tejido adiposo de manera más acelerada que en los hombres ${ }^{(19)}$.

El porcentaje de masa grasa aumentado se correlacionó positivamente de forma significativa con el IMC y el perímetro abdominal $>90 \mathrm{~cm}$; esto corrobora la eficacia de la medición del porcentaje de grasa corporal por DXA. En la mayoría de pacientes con síndrome metabólico, las variables IMC y perímetro abdominal se encuentran por encima de los límites normales, principalmente por la grasa visceral, contribuyendo al incremento del riesgo cardiovascular; sin embargo, existen casos en los cuales alguno de los componentes es normal mientras otros están elevados (ej.: IMC normal y perímetro abdominal y porcentaje de grasa elevados), por lo cual la combinación de todos permite una examen más completo.

A la exploración de la curva ROC, se encontró que el rendimiento del perímetro abdominal es solo $65 \%$ y el del IMC del 65,6\% para el diagnóstico de adiposidad. La superioridad de las mediciones del DXA para el diagnóstico de adiposidad se debe al hecho claramente establecido de que las mujeres tienen mayor cantidad de tejido adiposo (masa grasa), menos cantidad de músculo (masa magra) y una cantidad mayor de grasa en el tronco inferior y en la zona pélvica ${ }^{(20)}$, mientras que los hombres son capaces de almacenar mayor cantidad de grasa visceral y hepática ${ }^{(21)}$; por lo anterior, el perímetro abdominal y el IMC pueden variar ampliamente entre géneros aun cuando el porcentaje de masa grasa es el mismo ${ }^{(22)}$ y viceversa.
Uno de los hallazgos más importantes del estudio es el hecho de que el porcentaje de masa grasa mostró mayor riesgo que el IMC y el perímetro abdominal para enfermedad coronaria (OR: 1,9 p = 0,042). Estos hallazgos ya se habían demostrado en poblaciones europeas en el estudio de Lang et al. ${ }^{(23)}$, evidenciando que el porcentaje de masa grasa y magra son factores independientes determinantes de la salud, además de demostrar que el IMC predice desenlaces cardiovasculares desfavorables, pero solo con los valores más altos (sobrepeso y obesidad). Estos hallazgos son de particular interés, ya que en la práctica clínica existen pacientes con valores de IMC normales en los cuales la prevalencia de comorbilidades cardiovasculares y metabólicas, así como el riesgo de enfermedad coronaria, está lejos de ser despreciable ${ }^{(23-25)}$.

Se encontró una relación estadísticamente significativa entre el IMC aumentado y la hipertensión arterial (OR 3,0 $\mathrm{p}=0,033$ ), asociación que ya se había demostrado en el estudio multicéntrico INTERSALT, resultado de la interacción entre varios factores neurohormonales y de respuesta adrenérgica ${ }^{(26)}$.

Otros resultados fueron que la TMB disminuida se encuentra relacionada con la hipertensión arterial de forma significativa (OR: 1,70 p=0,046), concordante con otros estudios en los cuales los pacientes con mayor cantidad de masa muscular y TMB pueden asociarse a valores más bajos de tensión arterial $^{(27)}$ y que la variable IRME disminuida se relacionó con el aumento de las concentraciones séricas de LDL. En nuestro estudio, a pesar de que casi todos los pacientes tenían el porcentaje de masa magra normal (96,6\%), el 93,2\% de ellos presentaron la TMB disminuida, demostrando que a pesar de tener una cantidad adecuada de músculo esquelético, cursaban con un metabolismo basal bajo como resultado del desacondicionamiento, esta asociación se encuentra frecuentemente en la práctica clínica ${ }^{(9)}$ y puede estar explicada por la disminución en la capacidad del músculo de realizar un transporte de glucosa y síntesis de glucógeno efectivo, secundario tanto al desacondicionamiento como a la disminución de la masa magra total, promoviendo la estimulación de lipogénesis hepática de novo y contribuyendo al desarrollo de dislipidemia e hígado graso $^{(28)}$. Liu et $\mathrm{l}^{(29)}$ demostraron en una población China que aun cuando la ocurrencia del fenómeno de la resistencia a la insulina se debe al aumento de tejido adiposo, los cambios en el metabolismo del músculo esquelético y la cantidad de masa magra también juegan un papel crucial.

El IRME disminuido se relacionó con la falla cardiaca (OR $1,96 \mathrm{p}=0,078$ ), hallazgo coherente con estudios importantes como el de Narumi et $\mathrm{al}^{(30)}$, en el que se estableció que la presencia de sarcopenia se asocia a pronósticos desfavorables en la falla cardiaca y los valores más bajos de IRME se encontraron relacionados con peores clases funcionales por escala de NYHA y mayor cantidad de eventos cardiacos por análisis univariados de Cox. 
Una limitante de nuestro estudio es el hecho de que se realizó en enfermos del programa de rehabilitación cardiaca en prevención secundaria. Se deben realizar estudios prospectivos con población sana para determinar la asociación de riesgo entre los parámetros de composición corporal con enfermedad cardiovascular en nuestra población.

\section{Conclusiones}

El presente estudio demostró una asociación clara entre la adiposidad y la enfermedad coronaria, superior a la del IMC y el perímetro abdominal aumentado.

La suma de la medición de las variables antropométricas y de composición corporal por DXA ofrece información valiosa para el estudio y estimación del riesgo cardiovascular y metabólico de los pacientes.

La combinación de la medición de los parámetros antropométricos (perímetro abdominal e IMC) y de composición corporal (porcentaje de masa magra y grasa) es efectiva, dando la posibilidad de juzgar si el déficit o exceso de masa corporal es debido a una alteración en el porcentaje de masa grasa, masa magra o ambos.

Se requieren estudios de costoefectividad para recomendar la realización de DXA a todos los pacientes en prevención primaria o secundaria para la estimación del riesgo cardiovascular.

\section{Referencias}

1. Centers for Disease Control and Prevention. Obesity and overweight. 2006;(December 31, 2009). http://www.cdc.gov/nchs/fastats/overwt. htm.

2. Keys A, Fidanza F, Karvonen MJ, Kimura N, Taylor HL. Indices of relative weight and obesity.JChronic Dis. 1972;25(6-7):329-343. doi:10.1016/00219681(72)90027-6.

3. Pasco JA, Holloway KL, Dobbins AG, Kotowicz MA, Williams LJ, Brennan SL. Body mass index and measures of body fat for defining obesity and underweight: a cross-sectional, population-based study. BMC Obes. 2014;1(1):9. doi:10.1186/2052-9538-1-9.

4. Cornier M, Despres J, Davis N, et al. Assessing Adiposity: A Scientific Statement From the American Heart Association. Circulation. 2011;124(18):1996-2019. papers://d66c75de-80d3-4649-8e61-daeeb2a77aa9/Paper/p6293.

5. Cruz-Jentoft AJ, Baeyens JP, Bauer JM, et al. Sarcopenia: European consensus on definition and diagnosis: Report of the European Working Group on Sarcopenia in Older People. Age Ageing. 2010;39(4):412-423. doi:10.1093/ ageing/afq034.

6. IAEA Human Health Series No. 15. Dual Energy X Ray Absorptiometry for Bone Mineral Density and Body Composition Assessment.; 2010. http:// www-pub.iaea.org/books/iaeabooks/8459/Dual-Energy-X-Ray-Absorptiometry-for-Bone-Mineral-Density-and-Body-Composition-Assessment.

7. Albanese C V, Diessel E, Genant HK. Clinical applications of body composition measurements using DXA. J Clin Densitom. 2003;6(2):75-85. doi:JCD:6:2:75 [pii].

8. National Health and Nutrition Examination Survey (NHANES). Body Composition Procedures Manual. 2012:1-125.

9. Morrison DS, Boyle S, Morrison C, Allardice G, Greenlaw N, Forde L. Evaluation of the first phase of a specialist weight management programme in the UK National Health Service: prospective cohort study. Public Heal Nutr. 2012;15(1):28-38. doi:10.1017/S1368980011001625.

10. Weijs PJM, Vansant GAAM. Validity of predictive equations for resting energy expenditure in Belgian normal weight to morbid obese women. Clin Nutr. 2010;29(3):347-351. doi:10.1016/j.clnu.2009.09.009.

11. Buendía R, Zambrano M, Díaz Á, Reino A, Ramírez J, Espinosa E. Puntos de corte de perímetro de cintura para el diagnóstico de obesidad abdominal en población colombiana usando bioimpedanciometría como estándar de referencia. Rev Colomb Cardiol. 2015. doi:10.1016/j.rccar.2015.07.011.

12. Shea J, King M, Yi Y, Gulliver W, Sun G. Body fat Percentage is Associated with Cardiometabolic Dysregulation in BMI- Defined Normal Weight Subjects. Nutr Metab Cardiovasc Dis. 2011:1-7.

13. Sun Q Townsend MK, Okereke OI, Franco OH, Hu FB, Grodstein F. Adiposity and weight change in mid-life in relation to healthy survival after age 70 in women: prospective cohort study. BMJ. 2009;339(sep29_1):b3796. doi:10.1136/bmj.b3796.

14. De Lorenzo A, Del Gobbo V, Premrov MG, Bigioni M, Galvano F, Di Renzo L. Normal-weight obese syndrome: Early inflammation? Am J Clin Nutr. 2007;85(1):40-45. doi:85/1/40 [pii].

15. Pasco JA, Nicholson GC, Brennan SL, Kotowicz MA. Prevalence of Obesity and the Relationship between the Body Mass Index and Body Fat: CrossSectional, Population-Based Data. PLoS One. 2012;7(1):e29580 -
16. Perissinotto E, Pisent C, Sergi G, Grigoletto F. Anthropometric measurements in the elderly: age and gender differences. BrJ Nutr. 2002;87(2):177186. doi:10.1079/BJN2001487.

17. Vasconcelos FDAG, Cordeiro BA, Rech CR, Petroski EL. Sensibilidade e espe cificidade do índice de massa corporal no diagnóstico de sobrepeso / obesidade em idosos. Cad Saúde Pública. 2010;26(8):1519-1527.

18. Santos J., Albala C, Lera L, et al. Anthropometric measurements in the elderly population of Santiago, Chile. Nutrition. 2004;20(5):452-457. doi:10.1016/j.nut.2004.01.010.

19. Santosa S, Jensen MD. Adipocyte fatty acid storage factors enhance subcutaneous fat storage in postmenopausal women. Diabetes. 2013;62(3):775782. doi:10.2337/db12-0912.

20. Shen W, Punyanitya M, Silva AM, et al. Sexual dimorphism of adipose tissue distribution across the lifespan: a cross-sectional whole-body magnetic resonance imaging study. Nutr Metab (Lond). 2009;6:9. doi:10.1186/17437075-6-17.

21. Geer EB, Shen W. Gender differences in insulin resistance, body composition, and energy balance. Gend Med. 2009;6(Suppl 1):60-75. doi:10.1016/j. genm.2009.02.002.Gender

22. Silver HJ, Welch EB, Avison MJ, Niswender KD. Imaging body composition in obesity and weight loss: challenges and opportunities. Diabetes Metab Syndr Obes. 2010;3:337-347. doi:10.2147/DMSOTT.S9454.

23. Lang PO, Trivalle C, Vogel T, Proust J, Papazian JP. Markers of metabolic and cardiovascular health in adults: Comparative analysis of DEXA-based body composition components and BMI categories. J Cardiol. 2014;65(1):42-49. doi:10.1016/j.jjcc.2014.03.010.

24. Lang PO, Mahmoudi R, Novella JL, et al. Is obesity a marker of robustness in vulnerable hospitalized aged populations? Prospective, multicenter cohort study of 1306 acutely ill patients. J Nutr Heal Aging. 2014;18(1):66-74. doi:10.1007/s12603-013-0352-9.

25. Bradshaw PT, Monda KL, Stevens J. Metabolic syndrome in healthy obese, overweight, and normal weight individuals: the Atherosclerosis Risk in Communities Study. Obesity (Silver Spring). 2013;21(1):203-209. doi:10.1002/oby.20248.

26. Stamler J, Rose G, Stamler R, Elliott P. INTERSALT study findings. Public health and medical care implications. Circulation. 1989. http://hyper.ahajournals.org/content/14/5/570.short.

27. Vaziri Y, Bulduk S, Shadman Z, et al. Lean Body Mass as a Predictive Value of Hypertension in Young Adults, in Ankara, Turkey. Iran J Public Health. 2015;44(12):1643-1654. http://ovidsp.ovid.com/ovidweb.cgi?T=JS\&PAGE $=$ reference $\& D=$ prem $\& N E W S=N \& A N=26811815$.

28. Jornayvaz FR, Samuel VT, Shulman GI, Jornayvaz R. The Role of Muscle Insulin Resistance in the Pathogenesis of Atherogenic Dyslipidemia and Nonalcoholic Fatty Liver Disease Associated with the Metabolic Syndrome. Annu Rev Nutr. 2010;30(1):273-290. doi:10.1146/annurev.nutr.012809.104726.

29. Liu P, Ma F, Lou H, Liu Y. The utility of fat mass index vs. body mass index and percentage of body fat in the screening of metabolic syndrome. BMC Public Health. 2013;13(1):629. doi:10.1186/1471-2458-13-629.

30. Narumi $\mathrm{T}$, Watanabe $\mathrm{T}$, Kadowaki $\mathrm{S}$, et al Sarcopenia evaluated by fatfree mass index is an important prognostic factor in patients with chronic heart failure. Eur J Intern Med. 2015;26(2):118-122. doi:10.1016/j. ejim.2015.01.008. 